\title{
Atypical Presentation of Central Venous Occlusion (CVO) Disease
}

\author{
Rosnelifaizur $\mathrm{R}^{\star, \dagger, 1}$, Aizat Sabri I ${ }^{2}$, Krishna $\mathrm{K}^{3}$, Lenny $\mathrm{SS}^{4}$, Azim I ${ }^{5}, \mathrm{H}$ \\ Harunarashid ${ }^{6}$
}

\author{
${ }^{1}$ Department of Surgery, School of Medical Science, Universiti Sains Malaysia \\ ${ }^{2}$ Department of Surgery, School of Medical Science, Universiti Sains Malaysia \\ ${ }^{3}$ Department of Surgery, Faculty of Medicine, Universiti Kebangsaan Malaysia Medical Centre \\ ${ }_{4}^{4}$ Department of Surgery, Faculty of Medicine, Universiti Kebangsaan Malaysia Medical Centre \\ ${ }^{5}$ Department of Surgery, Faculty of Medicine, Universiti Kebangsaan Malaysia Medical Centre \\ ${ }^{6}$ Department of Surgery, Faculty of Medicine, Universiti Kebangsaan Malaysia Medical Centre \\ DOI: https://doi.org/10.15520/ijmhs.v9i1.2415
}

Accepted 4 Jan 2019; Received 2 Dec 2018; Publish Online 29 Jan 2019

Reviewed By: Dr.

Daniel V.

Department: Medical

\section{INTRODUCTION}

Chylothorax is an accumulation of lymph (containing a great amount of lymphocytes, triglycerides, and chylomicrons) in the pleura due to the blockage of thoracic duct drainage. The etiology may be from either traumatic or nontraumatic causes (sarcoidosis, neoplasm, chest irradiation, SVO etc), however there also have been reported as idiopathic forms. Direct trauma and malignancy are the most common causes of the chylothorax in adult with reported incidence of $50 \%$ and $30 \%$ respectively. However, the central vein thrombosis as a cause of chylothorax is uncommon and only a few cases has been reported in literature. It was mainly related with central venous catheterization. We reported a case of end stage renal failure (ESRF) patient with a previous history of central venous cannulation before and presented with persistent unilateral chylothorax.

\section{CASE REPORT:}

We reported a case of 58 years old gentleman who known case of end stage renal failure and had history of Right IJC cannulation of venous access on 2012, presented with recurrent shortness of breath, chesty cough and intermittent fever. Otherwise he got no hemoptysis, no recent contact with PTB patients and no joint pain. The cough he described as chesty, whitish sputum and intermittent The same presentation occurred last month with a pleural tapping was done and claimed it was a milky content. No further investigation was done at that moment. Current presentation he presented again with shortness of breath and intermittent coughing. Clincally noted a dullness in percussion up to midzone of right lung and reduce air entry on auscultation as well. Chest radiograph shows opacity in the right lung Figure 1. Pleural drain was inserted and the fluid was milky in nature. It was confirmed as a chylothorax with a present of cholesterol in a pleural fluid analysis. Computed tomography of the thorax Figure 2 showed complete occlusion of the superior vena cava with an established collateral circulation. LymphangiogramFigure 3 revealed lipiodol seen opacified lymph node and lymphatic vessels until the level of $\mathrm{T} 3$ on the right and T5 on the left. There was no obvious lipiodol opacification seen at the region of the right thorax. Effusion was improved after the instillation of fibrinolytic agent and the chest radiograph shows improvement.

\section{DISCUSSION:}

One of the complication of multiple central vein cannulation in ESRF patients can be a central venous occlusion. But it is rare to see the chylothorax as sequele from central venous occlusion. The pathogenesis it can be the direct impact or lesion to the thoracic duct opening and caused the high pressure in the system causing the rupture of thoracic duct and its collateral branch, hence it contribute to the chylothorax formation. In 1943, Blalock et al demonstrated that the interruption of the SVC will cause the chylothorax formation of $60 \%$ of cats and dogs [1] . The incidence has been reported occur in men secondary to spontaneous thrombosis or obstruction of the SVC, innominate vein, or subclavian vein. Other cases usually contributed by the placement of the cen- 


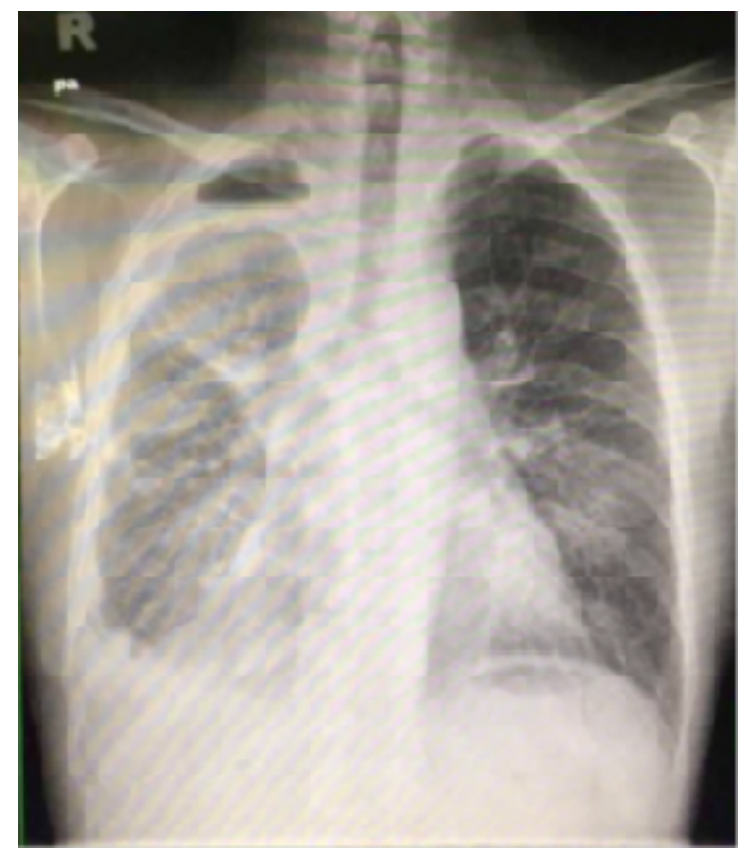

Figure 1. CXR shown Right pleural effusion

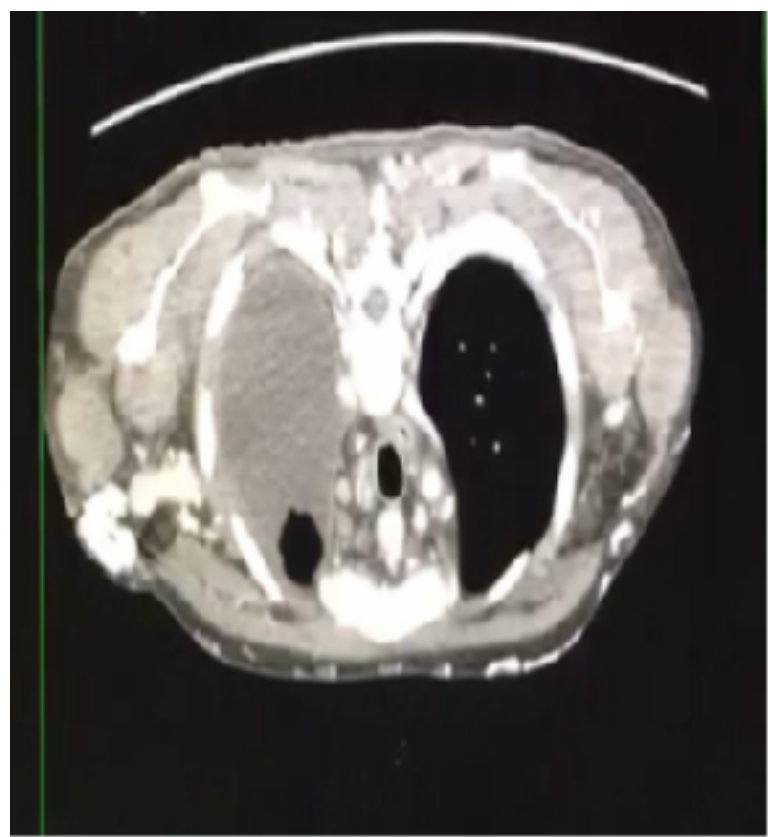

Figure 2. CECT Thorax shown Right SVC obstruction with right pleural Effusion

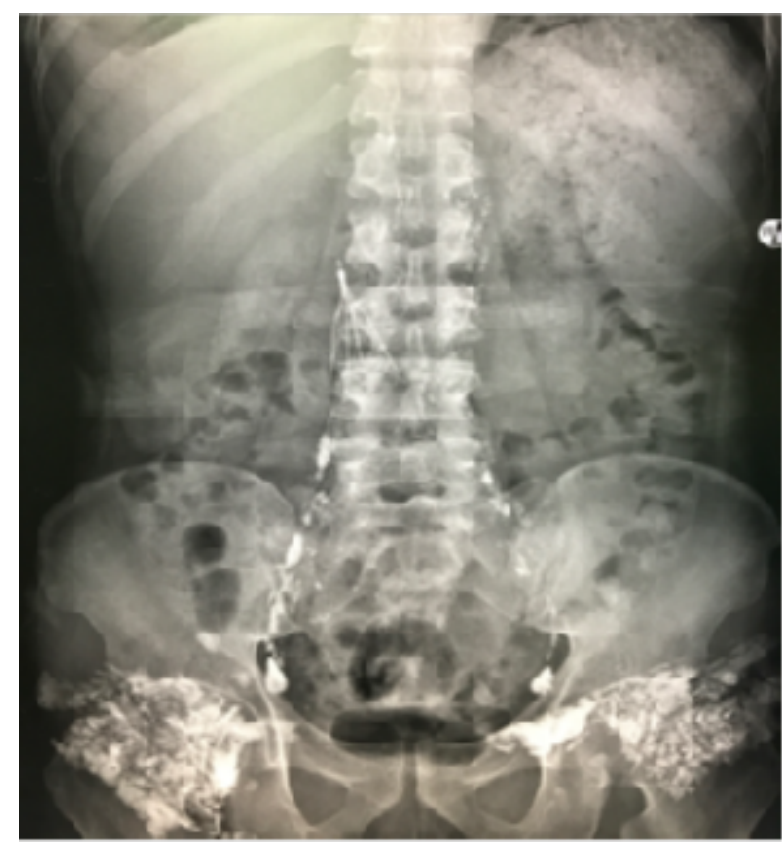

Figure 3. Lymphagiogram shown disruption

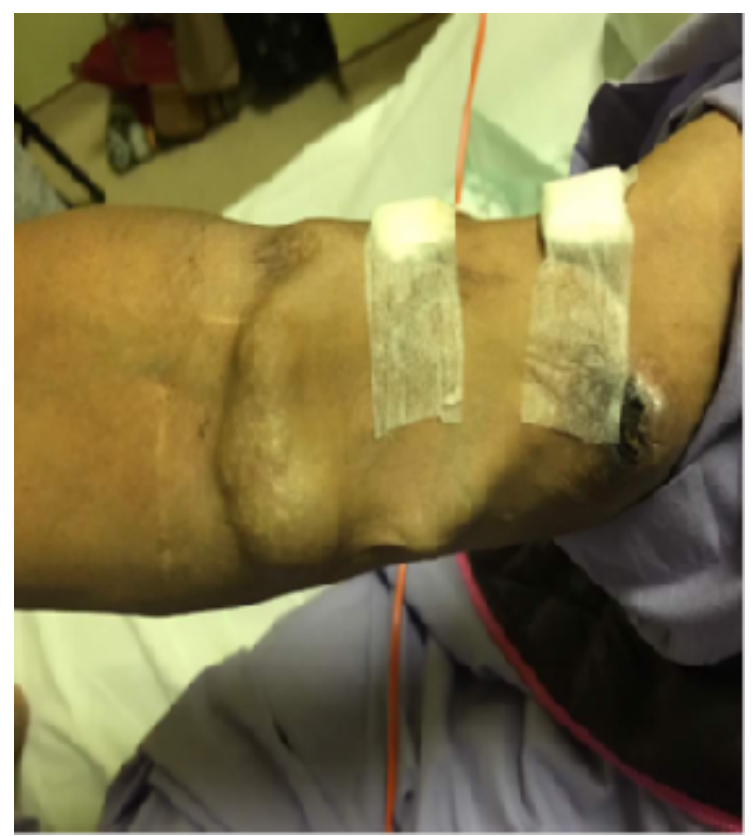

Figure 4. Right arm AVGof lymph flow cranially

tral venous catheter especially in newborn infants, children and adults [2] . The diagnosis is confirmed by the gross appearance of the pleural effusion, usually milky appearance and the composition of the fluid (triglycerides level higher than $110 \mathrm{mg} / \mathrm{dl}$ and elevated lymphocytes count) [2] [3] . The method of treatment of chylothorax in this situation is controversial. It depends on both of causes and symptoms. The approach is variety, can be from the conservative treatment to surgical intervention. Like in our patient, a low fat diet with medium chain triglycerides (that are di- 
rectly absrobed to blood) was introduced to decrease the amount of lymph. Chemical pleurodesis using tetracycline, bleomycin, or talc, and pleuroperitoneal shunts have been shown to be useful, in patients with vena cava obstruction, angioplasty may solve the problem [4] . Our patient, we decided to introduce the antifibrinolytic agent (streptokinase) to enhance the drainage of chylothorax, and to improve the symptoms. Minimal invasive surgery may be considered such as thoracoscopy with supradiaphragmatic repair or ligation of the thoracic duct may correct the chylothorax if other method has failed [5] . According to the previous reports, the syndrome of obstructed SVC with chylothorax has a poor prognosis $[4,5]$. Some have observed that, without relief of the venous obstruction, the lungs become lymphangiectatic themselves and this contributes to the long term morbidity [6] . We believe that early relief of the superior vena cava obstruction is important in the management of the chylothorax. Surgical intervention can be in the form of thrombectomy and reconstruction by using homograft patch can give a good result if it is done early [7]

\section{CONCLUSION:}

In conclusion, the presence of unilateral chylothorax with the previous history of central venous cannulation before should raise a high clinical index of suspicion among the clinicians to alert more about the presence of SVO and it should be considered in the differential diagnosis of nontraumatic chylous pleural By rapid diagnosis one could have done an early intervention pertaining to the SVC obstruction in order to reduce the morbidity and mortality due to the chylothorax.

\section{REFERENCES}

[1] Blalock A. Cunningham RS, Robinson CS.Experimental Production of Chylothorax by Occlusion of Superior Vena Cava,.Ann Surg. 1936 Sep;104(3):359-64;.

[2] Seibert JJ, Golladay ES, Keller C .Chylothorax secondary to superior vena caval obstruction.Pediatr Radiol; 1982.

[3] McGrath EE, Blades Z, Anderson PB. Chylothorax: aetiology, diagnosis and therapeutic options. Respir Med; 2010.

[4] Paul S, Altorki NK, Port JL, Stiles BM, Lee PC. Surgical management of chylothorax. Thorac Cardiovasc Surg 2009;57:226-8;.

[5] Kalra M, Gloviczki P, Andrews JC. Open surgical and endovascular treatment of superior vena cava syndrome caused by nonmalignant disease. J Vasc Surg;2003(38):215-23.

[6] PJ VV, S T. Chylothorax: a complication of a left subclavian vein thrombosis. Am J Clin Oncol;1996(19):99-101.

[7] Kalra M, Gloviczki P, Andrews JC. Open surgical and endovascular treatment of superior vena cava syndrome caused by nonmalignant disease. J Vasc Surg;2003(38):215-23.

\section{AUTHOR BIOGRAPHY}

Rosnelifaizur R Department of Surgery, School of Medical Science, Universiti Sains Malaysia

Aizat Sabri I Department of Surgery, School of Medical Science, Universiti Sains Malaysia

Krishna K Department of Surgery, Faculty of Medicine, Universiti Kebangsaan Malaysia Medical Centre

Lenny SS Department of Surgery, Faculty of Medicine, Universiti Kebangsaan Malaysia Medical Centre

Azim I Department of Surgery, Faculty of Medicine, Universiti Kebangsaan Malaysia Medical Centre

H Harunarashid Department of Surgery, Faculty of Medicine, Universiti Kebangsaan Malaysia Medical Centre 